\title{
PAPER
}

\section{The development of route learning in Down syndrome, Williams syndrome and typical development: investigations with virtual environments}

\section{Harry R.M. Purser, ${ }^{1}$ Emily K. Farran, ${ }^{2}$ Yannick Courbois, ${ }^{3}$ Axelle Lemahieu, ${ }^{3}$ Pascal Sockeel, ${ }^{3}$ Daniel Mellier ${ }^{4}$ and Mark Blades ${ }^{5}$}

1. School of Psychology, University of Nottingham, UK

2. Department of Psychology and Human Development, Institute of Education, University of London, UK

3. Laboratoire PSITEC (EA 4072), UDL3, Université Lille Nord de France, France

4. Laboratoire PSY-NCA (EA 4306), Université de Rouen, France

5. Department of Psychology, University of Sheffield, UK

\begin{abstract}
The ability to navigate new environments has a significant impact on the daily life and independence of people with learning difficulties. The aims of this study were to investigate the development of route learning in Down syndrome ( $\mathrm{N}=50)$, Williams syndrome $(\mathrm{N}=19)$, and typically developing children between 5 and 11 years old $(\mathrm{N}=108)$; to investigate use of landmarks; and to relate cognitive functions to route-learning ability in these groups. Overall, measures of attention and long-term memory were strongly associated with route learning, even once non-verbal ability was controlled for. All of the groups, including 5- to 6-year-old TD children, demonstrated the ability to make use of all landmark types to aid route learning; those near junctions, those further from junctions, and also distant landmarks (e.g. church spire, radio mast). Individuals with WS performed better than a matched subset of TD children on more difficult routes; we suggest that this is supported by relatively strong visual feature recognition in the disorder. Participants with DS who had relatively high levels of non-verbal ability performed at a similar level to TD participants.
\end{abstract}

\section{Research highlights}

- Individuals with Williams syndrome performed better than a matched subset of typically developing children on more difficult routes.

- Measures of attention and long-term memory were strongly associated with route learning.

- All of the groups, including 5- to 6-year-old typically developing children, demonstrated the ability to make use of various landmark types to aid route learning, including distant landmarks.

\section{Introduction}

Spatial knowledge of novel environments is typically gained by moving through those environments. Siegel and White (1975) proposed that the development of spatial knowledge proceeds via three distinct stages, each a precursor of the next: landmark knowledge, which can be described as information about objects without knowledge of the relations between them; route knowledge, which is characterized as knowledge of the sequence of landmarks and turns along a path of a particular route; and configural knowledge, which is information about the layout of an environment that includes distance and direction between landmarks. Although recent evidence does not support the hierarchical nature of this model (e.g. Montello, 1998; Ishikawa \& Montello, 2006), these three categories of knowledge are nonetheless relevant to studies of spatial navigation. In typical adults, the model is broadly supported at a neural level; although part of an interacting network, the parahippocampal gyrus shows activation to landmarks along a route (Wegman \& Janzen, 2011), whilst

Address for correspondence: Harry R.M. Purser, School of Psychology, University Park, University of Nottingham, Nottingham NG7 2RD, UK; e-mail: harry.purser@nottingham.ac.uk

The copyright line for this article was changed on November 14, 2014 after original online publication. 
route knowledge is associated with the caudate nucleus, and the hippocampus shows activation for configural knowledge of the environment (Doeller, King \& Burgess, 2008). The current study explored the first two stages, landmark knowledge and route knowledge: its aims were to chart the development of route-learning ability and landmark use in typically developing (TD) children, individuals with Down syndrome (DS) and individuals with Williams syndrome (WS), and to determine the cognitive correlates of route learning in each group, i.e. verbal ability, non-verbal ability, attention, and memory.

\section{Cognitive profiles of Williams and Down syndromes}

Down syndrome (DS) is a neurodevelopmental disorder with a prevalence of $\sim 5$ in 10,000 (Steele \& Stratford, 1995) and results from an extra copy, or part-copy, of chromosome 21. Individuals with DS generally present difficulties with verbal tasks (e.g. Chapman, 1995) but less impairment of visuo-spatial ability (e.g. Purser \& Jarrold, 2005). Williams syndrome (WS) is a neurodevelopmental disorder, with a prevalence of $\sim 1$ in 20,000 (Morris, Dempsey, Leonard, Dilts \& Blackburn, 1988), resulting from a microdeletion of 28 genes from one copy of chromosome 7 (Ewart, Morris, Atkinson, Jin, Sternes, Spallone, Stock, Leppert \& Keating, 1993). The disorder is characterized by a cognitive profile that contrasts with the one seen in DS: despite marked difficulties with visuo-spatial cognition, verbal abilities are less impaired in individuals with WS (e.g. Howlin, Davies \& Udwin, 1998). For both DS and WS groups, level of ability also varies within each domain, on account of differences in task demands and the associated neural systems. For example, visuo-spatial perception is typically stronger than visuo-motor performance in WS (Farran, Jarrold \& Gathercole, 2003); and performance on the CANTAB Paired Associate Learning task (requiring participants to learn and remember abstract visual patterns associated with various locations) is an impaired aspect of visuo-spatial cognition in DS (e.g. Pennington, Moon, Edgin, Stedron \& Nadel, 2003; Edgin, Mason, Allman, Capone, DeLeon, Maslen, Reeves, Sherman \& Nadel, 2010). Thus, whilst we cannot predict the relative levels of ability on our environmental learning tasks in DS and in WS, given the broad differing cognitive profiles, it is possible that any difficulty in environmental learning experienced by each population will be a product of different underlying deficits.

\section{Environmental learning in Williams and Down syndromes}

For both DS and WS, research into cortical structure or activation has focused on predetermined regions of interest. Thus, whilst there is evidence for atypicalities of the hippocampus in both groups (DS: Pinter, Brown, Eliez, Schmitt, Capone \& Reiss, 2001; WS: MeyerLindenberg, Hariri, Munoz, Mervis, Mattay, Morris \& Berman, 2005), activation of the caudate nucleus and parahippocampal gyrus have not been investigated. Behavioural and neural evidence (when available) is discussed below.

Pennington and colleagues (Pennington et al., 2003) investigated the performance of adolescents with DS on a virtual version of the Morris water maze (Morris, 1981). The participants with DS performed less well than mental-age-matched controls. However, a larger study by Edgin et al. (2010) did not find any impairment in DS on the same task, relative to mental-age-matched controls, although Edgin and colleagues interpreted the overall pattern of visuo-spatial behaviours observed in DS as a hippocampal deficit. This is consistent with evidence of a small hippocampus in DS, relative to overall brain volume (Pinter et al., 2001). Also in line with the notion of a hippocampal deficit, decreased neurogenesis has been demonstrated in the dentate gyrus of the hippocampus in both foetuses with DS and Ts65Dn mice (Contestabile, Fila, Ceccarelli, Bonasoni, Bonapace, Santini, Bartesaghi \& Ciani, 2007), indicating a plausible mechanism for some of the visuo-spatial problems associated with the disorder. There are four recent studies of environmental learning in WS. In a real-world study Farran, Blades, Boucher and Tranter (2010) found that their WS group were worse at retracing a route than a group with moderate learning difficulties matched for mental and chronological age (and were also worse than a chronological-age-matched TD group). However, the WS group showed a similar degree of improvement in following the correct route as the control groups across learning trials.

Building on this work, Farran and colleagues (Farran, Courbois, Van Herwegen \& Blades, 2012a; Farran, Courbois, Van Herwegen, Cruickshank \& Blades, 2012b) assessed route learning and recall of landmarks in WS and typical development, using virtual environments (VEs). Farran et al. (2012a) used VEs of brick-wall mazes with six junctions, each featuring 16 landmarks. Half of the landmarks on the correct path through the maze were close to junctions (junction landmarks); the other half were not (path landmarks). The participants with WS made more route-learning errors than 9-year-old TD participants. This performance impairment was primarily due to perseverative errors (errors made at the same junction on consecutive learning trials) on the part of the WS group, consistent with other evidence of an executive dysfunction in this population (e.g. Menghini, Addona, Costanzo \& Vicari, 
2010). The TD group showed superior recall of junction landmarks over path landmarks. This recall advantage for junction landmarks was also shown by participants with WS, but only for those with higher non-verbal ability. Poor route knowledge, as well as configural knowledge, in WS has been further demonstrated using a VE cross-maze (a square maze with four radial arms), where individuals with WS required more trials to learn a route than TD 10-year-olds (Broadbent, Farran \& Tolmie, in press). Neural evidence to date relates to configural knowledge only; hippocampal activation and structure is impaired in WS (Meyer-Lindenberg et al., 2005). Furthermore, a link between hippocampal deficits and spatial learning has been demonstrated in LIMK-1 knockout mice (LIMK-1 is deleted in WS) (Meng, Zhang, Tregoubov, Janus, Cruz, Jackson, Lu, MacDonald, Wang, Falls \& Jia, 2002). In humans, however, the contribution of LIMK-1 to spatial learning in WS is likely in combination with other deleted genes in the WS critical region (Gray, Karmiloff-Smith, Funnell \& Tassabehji, 2006).

\section{Landmark use in typical development}

The dissociation between junction and path landmarks discussed above has been shown in TD adults at both behavioural and neural levels. That is, activation of the parahippocampal gyrus (PHG) is higher for landmarks at decision points (junction landmarks) than landmarks at non-decision points (path landmarks) and higher PHG activation is correlated with stronger recognition of landmarks (Wegman \& Janzen, 2011). Whilst we are not aware of research into the neural correlates of landmark use in TD children, behavioural evidence demonstrates that the preferential use of junction over path landmarks emerges with development. That is, when asked to recall landmarks along a learnt route TD children as young as 6 years can encode junction landmarks better than path landmarks (Farran et al., 2012a).

Although many studies have been concerned with landmarks on routes, few have addressed the utility of distant landmarks for children, such as church spires. Cornell, Heth and Broda (1989) found that 12-year-olds, but not 6-year-olds, benefit from being told to pay attention to distant landmarks during route learning. As noted above, there is mixed evidence for whether people with DS are less good than mental-age-matched controls at using distant landmarks in a virtual Morris maze (Pennington et al., 2003; Edgin et al., 2010). Individuals with WS were faced with distant landmarks only in Broadbent et al. (in press) and, as discussed, demonstrated route knowledge below a typical 10-year-old level.
One final point concerning landmarks is that their proficient use must depend on long-term visual recognition abilities. Jarrold, Baddeley and Phillips (2007) assessed long-term memory among participants with DS and WS, using the Doors and People battery (Baddeley, Emslie \& Nimmo-Smith, 1984), which provides measures of both long-term recall and recognition of both verbal and visual information. When scores were standardized on non-verbal mental age relative to a typically developing sample, neither disorder group was impaired on long-term visual recognition. This alleviates (but does not preclude) possible concerns that differences in landmark-related behaviour could be a consequence of visual recognition problems.

\section{Overview of the current study}

Experiment 1 investigated the development of route learning using a VE maze that featured a variety of landmarks. Experiment 2 assessed use of junction, path and distant landmarks by running three different mazes for each participant, each featuring one of these landmark types in isolation.

\section{Predictions}

The different cognitive profiles of DS and WS were expected to give rise to differing patterns of performance in Experiment 1. In the absence of evidence regarding parahippacampal gyrus or caudate nucleus function in these groups, predictions based on neural evidence can only be tentative; evidence of impaired hippocampal function in both groups could indirectly impact landmark knowledge and route knowledge, on account of connections within the environmental learning neural network. Based on behavioural evidence, the WS group was expected to perform at or below their general level of visuo-spatial ability at route learning, which would represent a weakness within their cognitive profile. For the DS group, behavioural evidence for impaired configural knowledge demonstrates that environmental learning might not follow the cognitive profile of relatively strong visuo-spatial performance in this group. However, given the absence of research into landmark or route knowledge in this group, strong predictions could not be made.

We predicted differences in the underlying mechanisms that drive route-learning performance across groups. To this end, a cognitive battery was administered including measures of executive control and long-term memory, which are known to influence route learning in TD children (Purser, Farran, Courbois, Lemahieu, Sockeel \& Blades, 2012). 


\section{Developmental trajectories}

The current study concerns neurodevelopmental disorders; the route-learning data were therefore analysed within a developmental framework. A developmental trajectories approach was adopted (Thomas, Annaz, Ansari, Scerif, Jarrold \& Karmiloff-Smith, 2009), in which functions of task performance against mental age are constructed, which allow the comparison of developmental change across groups. Such trajectories can identify developmental differences between participant groups that simple group-matched designs cannot reveal.

\section{Experiment 1 Method}

\section{Participants}

Participants were 108 TD individuals, 50 individuals with a clinical diagnosis of DS, and 19 individuals with a clinical diagnosis of WS. The WS group, 67 of the TD group and 21 DS group were English, the remainder were French. The DS group included three participants over the age of 30; although there is some concern about such participants being at risk of Alzheimer-like dementia (e.g. Lott \& Head, 2001), all subsequent analyses were repeated with and without these participants and no differences were evident between these analyses. All participants with WS had been diagnosed based on phenotypic and genetic information. Genetic diagnosis was based on a Fluorescent in situ Hybridization (FISH) test (see Lenhoff, Wang, Greenberg \& Bellugi, 1997). English participants were tested on the British Picture Vocabulary Scale III (BPVS; Dunn, Dunn, Styles \& Sewell, 2009); the French participants were tested on the equivalent, L'échelle de vocabulaire en images Peabody (EVIP; Dunn, Theriault-Whalen \& Dunn, 1993). For brevity, both tests will be referred to as BPVS henceforth. Participants were also assessed on Raven's Coloured Progressive Matrices (RCPM; Raven, Raven \& Court, 1998). Participant information is given in Table 1.

\section{Maze task}

A virtual environment (VE) maze, created using Virtools 5.0, was used. The maze consisted of a network of roads at right angles to each other, lined with brick walls. There were six junctions, each leading to one correct and one incorrect path section. Across these junctions, there were two left, two right, and two straight-ahead choices that led to the next correct path segment. Correct choices were balanced by the equivalent type and number of incorrect choices. Incorrect path choices ended in a cul-de-sac, which had the same appearance as a
Table 1 (a). Descriptive statistics for route learning and the cognitive test battery; (b). Scores for route learning, Go/No Go and the People Test

\begin{tabular}{|c|c|c|c|}
\hline & Mean & $S D$ & Range \\
\hline \multicolumn{4}{|l|}{ (a) } \\
\hline \multicolumn{4}{|c|}{ Age (years;months) } \\
\hline TD & $8 ; 4$ & $1 ; 9$ & $5 ; 4-11 ; 4$ \\
\hline DS (Expt 1) & $18 ; 7$ & $7 ; 4$ & $10 ; 2-39 ; 1$ \\
\hline DS (Expt 2) & $18 ; 3$ & $6 ; 1$ & $10 ; 2-39 ; 1$ \\
\hline WS & $20 ; 2$ & $7 ; 4$ & $12 ; 1-30 ; 7$ \\
\hline \multicolumn{4}{|l|}{ BPVS (raw) } \\
\hline TD & 108 & 25 & $41-150$ \\
\hline DS (Expt 1) & 81 & 23 & $27-143$ \\
\hline DS (Expt 2) & 80 & 22 & $27-142$ \\
\hline WS & 116 & 22 & 79-158 \\
\hline \multicolumn{4}{|l|}{ RCPM (raw) } \\
\hline TD & 27 & 5.6 & $9-36$ \\
\hline DS (Expt 1) & 17 & 4.8 & $9-31$ \\
\hline DS (Expt 2) & 17 & 4.9 & $9-31$ \\
\hline WS & 19 & 4.6 & $12-29$ \\
\hline \multicolumn{4}{|l|}{ (b) } \\
\hline \multicolumn{4}{|c|}{ Route learning errors } \\
\hline \multicolumn{4}{|c|}{ Maze 1} \\
\hline TD & 5.1 & 7.7 & $0-37$ \\
\hline DS & 17.6 & 16.2 & $0-72$ \\
\hline WS & 4.7 & 7.7 & $0-28$ \\
\hline \multicolumn{4}{|c|}{ Maze 2A: Junction landmarks } \\
\hline TD & 1.2 & 2.6 & $0-14$ \\
\hline DS & 6.0 & 8.1 & $0-36$ \\
\hline WS & 3.2 & 4.7 & $0-15$ \\
\hline \multicolumn{4}{|c|}{ Maze 2A: Path landmarks } \\
\hline TD & 1.6 & 3.5 & $0-27$ \\
\hline DS & 8.8 & 10.4 & $0-39$ \\
\hline WS & 2.6 & 3.6 & $0-13$ \\
\hline \multicolumn{4}{|c|}{ Maze 2A: Distant landmarks } \\
\hline TD & 8.6 & 10.9 & $0-56$ \\
\hline DS & 11.9 & 10.3 & $0-41$ \\
\hline WS & 7.6 & 9.1 & $0-29$ \\
\hline \multicolumn{4}{|c|}{ Go/No Go RT (ms) } \\
\hline TD & 630 & 126 & $387-1031$ \\
\hline DS & 722 & 188 & $420-1264$ \\
\hline WS & 768 & 120 & $607-998$ \\
\hline \multicolumn{4}{|c|}{ Go/No Go Errors } \\
\hline TD & 3.9 & 2.9 & $0-15$ \\
\hline DS & 5.4 & 6.5 & $0-26$ \\
\hline WS & 4.1 & 4.4 & $0-16$ \\
\hline \multicolumn{4}{|l|}{ People Test } \\
\hline TD & 19.6 & 9.5 & $0-36$ \\
\hline DS & 11.8 & 9.1 & $0-32$ \\
\hline WS & 16.7 & 8.9 & $0-31$ \\
\hline
\end{tabular}

Note: $\mathrm{TD}=$ typically developing, $\mathrm{DS}=$ Down syndrome, $\mathrm{WS}=$ Williams syndrome, BPVS = British Picture Vocabulary Scale, RCPM = Raven's Coloured Progressive Matrices.

T-junction when viewed from the preceding junction. A map of the maze layout is shown in Figure 1.

Thirty-two landmarks were placed in or around the maze (see Figures 1 and 2). Landmark objects were selected from a range of categories (e.g. animals, tools, furniture) and were chosen for high verbal frequency (Morrison, Chappell \& Ellis, 1997) and also for being easy to recognize. There were 19 unique landmarks: eight 


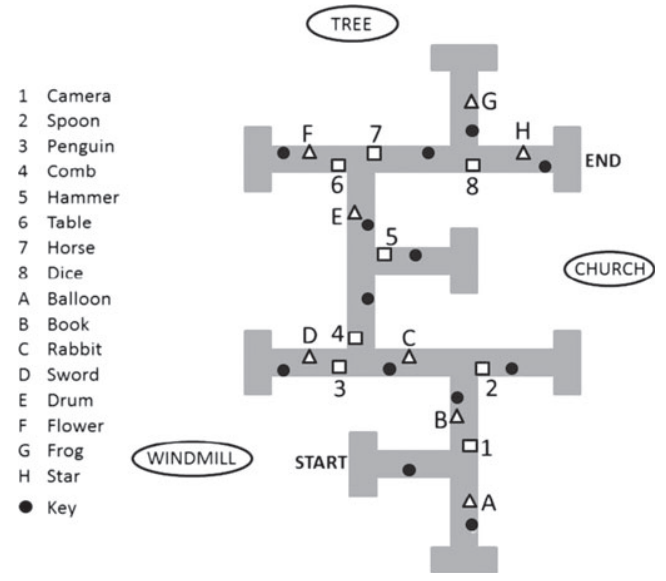

Figure 1 A map of the maze used in Experiment 1. Note: 1 to 8 are junction landmarks and $A$ to $H$ are path (nonjunction) landmarks. The black circles are non-unique landmarks (keys). Distant landmarks (tree, church, windmill) are outside the maze walls.

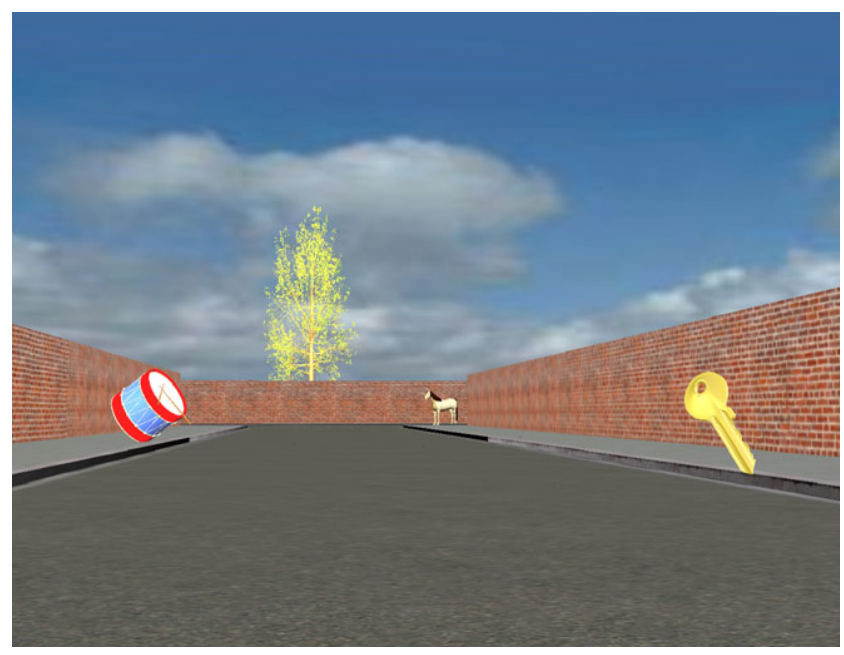

Figure 2 The virtual environment maze in Experiment 1 Note: Shows example landmarks: drum and horse (junction landmarks, key (non-unique landmark) and the tree (distant landmark).

of the unique landmarks were near to junctions ('junction landmarks'). Eight of the unique landmarks were not near to junctions ('path landmarks'). There were three unique landmarks outside the maze ('distant landmarks'). Each of the three distant landmarks was visible from most locations within the maze, though it was not possible for all three to always be visible, given the height of walls necessary for the maze to be effective (such that the maze solution was not visible to participants). Landmarks within the maze were equally distributed to the left and right of the path. There were
13 non-unique landmarks: the same landmark, a key, appeared once on each of the 13 path segments.

At the end of the maze was a metallic ball, which elicited the words 'Well done!' and a fanfare sound when the participant reached it.

\section{Procedure}

Each participant was first familiarized with how to manually control their navigation through a VE by completing a short familiarization maze with no landmarks. Participants followed a single path, which included two right-angle turns; there were no decisions to be made. Movement through the maze was controlled by computer keyboard and mouse: the space bar caused forwards movement, while orientation was controlled by the mouse.

After the familiarization maze, the experimenter showed the participant the correct route through the experimental maze, saying to the participant, 'Pay close attention to the route and also to the various objects that appear in the "maze game" because you will have to go exactly the same way through the maze after I have shown you.' The participant then attempted to navigate through the maze, with a maximum of 10 trials to complete the maze from start to finish without error on two consecutive trials. Because the incorrect path sections ended in cul-de-sacs, when the participant made an error, they were able to self-correct; encouragement was given, but no help. Each trial terminated on completion of the route.

The dependent variable was the total number of errors made across all trials, henceforth referred to as Maze Error. An error was defined as a deliberate incursion down an incorrect path; if the participant corrected his/ her course before reaching half-way down an incorrect path section, no error was counted. The computer program automatically detected errors.

After participants had completed the above task they were asked to complete the two-turn familiarization maze once more, as quickly as possible. This served as a measure of keyboard/mouse proficiency.

A naming task was then administered. To ensure that the landmarks used could be identified, participants were shown images of each of the 20 landmarks in a pseudorandom order and were asked to name them.

\section{Cognitive test battery}

\section{Go/No Go (GNG) task}

GNG was chosen as a measure of executive control. A pseudo-random series of red, green, blue and yellow solid circles (diameter: $5 \mathrm{~cm}$ ) was presented on a computer. Participants were instructed to press the space 
bar as rapidly as possible when they saw each circle, unless it was red, in which case they should refrain from pressing the space bar. If the space bar was pressed on red, a buzzing 'error' noise was heard and the circle disappeared. Each circle disappeared after 2 seconds if the space bar was not pressed. If participants pressed the space bar on two consecutive red trials, they were reminded of the task rules. There were eight practice trials, followed by 128 experimental trials, with a break after 64. The dependent measures were the average reaction time (RT) for correct hits and the number of errors (pressing the space bar for a red circle).

\section{People Test}

The People Test (Baddeley et al., 1994) is a measure of long-term verbal memory. Participants were shown pictures of four people in succession, each paired with a forename and surname (Jim Green, Cuthbert Cattermole, Tom Webster, Philip Armstrong) which were printed under the picture. The experimenter told the participant the occupation of each person and said his name aloud (e.g. 'This is the doctor. His name is Jim Green'), before moving on to the next person. Immediately after being shown all four people and told their professions, there was a test phase: for each person in turn, participants were cued with the profession of the person encountered and asked to produce the relevant name ('Can you tell me the name of the doctor?'). This test phase was repeated two more times, or until perfect performance was attained, in which case a maximum score was assumed for the remaining trials. Each forename or surname recalled correctly earned one point and one additional point was granted for successfully recalling a forename and surname together (one point for 'Jim Heath' or 'Tom Green', 3 points for 'Jim Green'). The maximum score was 36 ( 3 points for each name $\times 4$ names $\times 3$ trials).

\section{Experiment 1 Results}

Table 1 shows descriptive statistics for route-learning performance and for the items in the cognitive test battery.
The majority of participants required fewer than 10 learning trials to learn the route (TD: $96 \%$; DS: $72 \%$; WS: $89 \%)$ and overall the TD and WS groups each made a mean of 5 route-learning errors (Maze Error), whereas the DS group made 18 , a significant group difference, $F(2$, $174)=24.343, p<.001, \eta_{p}{ }^{2}=219$ (DS < WS, TD). Considered separately, the English and French TD samples showed the same patterns of correlations between Maze Error and the other measures, with the exception of Go/No Go: Go/No Go RT was reliably associated with Maze Error for the UK TD sample, but Go/No Go error was not. Neither was associated with Maze Error in the French TD sample. Given that the UK pattern prevailed when participants were treated as one group, this lack of relationships in the French sample is likely due to noise.

Correlations between the test battery items and Maze Error are displayed in Table 2. The distribution of Maze Error was positively skewed in the WS group, so Spearman's $r$ ho is reported instead of Pearson's $r$ for this group.

The relationships between test battery items and maze error were examined further with partial correlations, controlling for RCPM to 'factor out' variance associated with general fluid intelligence (i.e. controlling for noncentral task demands; see Jarrold \& Brock, 2004), thus giving a stricter assessment of possible relationships between our background measures and route learning. For the TD group, only Go/No Go RT, $r(102)=.247, p<$ .05 , and People, $r(102)=-.368, p<.001$, remained reliably associated with route-learning performance. For the DS group, Go/No Go Error was significantly associated with route learning, $r(39)=.354, p<.05$, and the association with People and route learning was marginally significant, $r(39)=-.279, p=.078$. Partial correlations could not be run for the WS group because of the skew in Maze Error.

\section{Trajectory analyses}

RCPM was strongly related to route-learning performance in all three groups and was continuously distributed, indicating that a trajectory design was appropriate (Thomas et al., 2009). However, a large proportion of the WS group made no Maze Error; this

Table 2 Correlations between cognitive measures and route-learning errors in Experiment 1

\begin{tabular}{|c|c|c|c|c|c|c|c|}
\hline & Age & BPVS & RCPM & GNG RT & GNG errors & People & Computer \\
\hline TD & $-.50 * *$ & $-.41 * *$ & $-.50 * *$ & $.42 * *$ & .06 & $-.57 * *$ & -.03 \\
\hline $\mathrm{DS}$ & -.13 & $-.37 * *$ & $-.45^{* *}$ & .22 & $.37 * *$ & $-.34 *$ & .10 \\
\hline WS (non-parametric) & -.20 & $-.68 * *$ & $-.58 * *$ & -.13 & $.73^{* *}$ & -.45 & .26 \\
\hline
\end{tabular}

Note: $\mathrm{BPVS}=$ British Picture Vocabulary Scale, RCPM = Raven's Coloured Progressive Matrices, GNG RT = Go/No Go task reaction time,

Computer $=$ a measure of mouse/keyboard and eye coordination. ${ }^{*} p<.05 ;{ }^{*} p<.01$. 
made these data unsuitable for such analyses and they will be considered separately below. The trajectory analyses were univariate ANCOVAs with group as the between-subjects factor and RCPM as the covariate. The ANCOVA model included interaction terms between the RCPM covariate and the between-subjects factor, to explore whether route-learning performance developed at a different rate in each group with respect to nonverbal ability. The data were analysed with respect to RCPM (non-verbal ability), rather than chronological age, because chronological age was not associated with task performance in the DS (or WS) group.

There was a steeper relationship between RCPM and Maze Error in the DS group than the TD group, $F(1$, $154)=5.563, p<.05, \eta_{p}{ }^{2}=.035$ (Figure 3), and at the intercept the DS group's Maze Errors were significantly higher than the TD group's, $F(1,154)=7.378, p<.01$, $\eta_{p}{ }^{2}=.046$. Put together, low non-verbal ability was more disruptive to route-learning performance in the DS group than in the TD group and the group difference reduced as RCPM score increased.

The WS group was individually matched on RCPM raw score to 19 TD children. The WS group made significantly fewer Maze Errors than this TD group, indicated by a nonparametric Mann-Whitney U-test, $U(38)=95.50, p=.012$.

\section{Experiment 1 Discussion}

There were two aims of Experiment 1: the first was to explore which factors determine route-learning

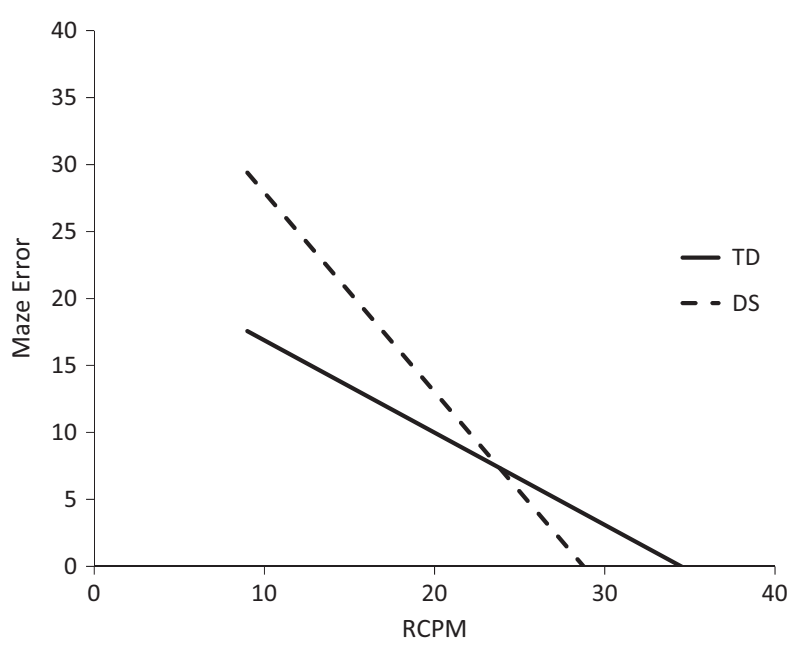

Figure 3 Experiment 1: Maze Error against RCPM score for the TD and DS groups.

Note: $T D=$ typically developing, DS = Down syndrome, $R C P M=$ Raven's Coloured Progressive Matrices. performance in each group; the second was to explore the level of route knowledge of the DS and WS groups relative to the TD group. Surprisingly, the DS group demonstrated the poorest route-learning ability. Trajectory analysis unpacked this finding further. In contrast, the WS sample performed above the level predicted by non-verbal ability. Correlations showed that both verbal and non-verbal ability predicted route-learning performance in all three groups. However, age predicted performance only in the TD group, indicating that ability is much less closely related to age in developmental disorders (as is often the case, see KarmiloffSmith, 1998).

\section{Cognitive correlates of route learning}

For the TD group, Go/No Go RT, but not Go/No Go Error, predicted route learning; for the DS and WS groups, route learning was predicted by Go/No Go Error, but not Go/No Go RT. This may reflect different aspects of attention being important for the groups: reaction time reflects the general attentional resources (and also processing speed) involved in concentrating on a task, with higher speed perhaps associated with better sustained attention (e.g. Redick, Calvo, Gay \& Engle, 2011). In contrast, errors result from impulsive responding, or a lack of motor-control inhibition. Thus, perhaps sustained attention led to more route-learning errors in the TD group, whereas poorer inhibition was associated with more errors in the disorder groups.

Long-term memory was associated with route learning in the TD and DS groups, but not the WS group. Several participants with WS made no errors; this lack of performance variability meant that their performance could not be correlated to other variables. Certainly, each group must have made use of long-term memory in the route-learning task in order to remember which way to turn at junctions, and/or to recognize landmarks. Given the very strong association of Go/No Go Error for the WS group, we infer that errors were made by this group because of poor attentional inhibition, but not because of forgetting the route.

Computer Control score, our measure of keyboard/ mouse proficiency, was not associated with route-learning performance in any group. This was important to establish, because any such association could have masked other relationships between variables.

General cognitive ability would be expected to contribute to task performance in terms of non-central task demands. Even after controlling for such ability, as indexed by RCPM, the associations between routelearning error and attention and between route-learning error and long-term memory remained strong in the TD 
and the DS groups (the WS data were not suited to this analysis).

\section{Developmental trajectories of route learning}

The trajectory analyses revealed that higher RCPM scores were associated with superior route-learning ability in both groups, but that low non-verbal ability had more of a negative impact on route-learning performance in the DS group than in the TD group, deviating somewhat from the prediction that the DS group would perform in line with non-verbal ability. However, the DS group's performance approaches that of the TD group at higher non-verbal ability levels: it 'catches up'. Thus, route-learning ability is mental-ageappropriate for higher-functioning individuals with DS, but below the level predicted by mental age for lowerfunctioning individuals.

The WS group was individually matched on nonverbal ability to a sample of TD children. The WS group performed better at the route-learning task than the matched TD group. It is unusual to find superior ability in matched developmental disorder samples. This suggests that route knowledge, although not at a chronological age appropriate level, represented a relative peak within the non-verbal domain for this group. This pattern contrasts with Farran et al. (2012a), whose WS group made a comparable number of errors to TD 6-, 7and 8-year-old children, and more route-learning errors than 9-year-old TD participants. Given that non-verbal mental age is typically at about the 6-year-old level in WS, the participants in Farran et al. (2012a) performed within the range expected for TD children of the same non-verbal mental age to slightly above that level. This slight difference between studies is not related to increased cognitive variability in WS as the WS groups used across the two studies are largely overlapping. Equally, cognitive variation in WS has been shown to be no more wide-ranging than the typical population with reference to verbal, non-verbal and working memory measures (Van Herwegen, Rundblad, Davelaar \& Annaz, 2011). The difference is most likely a reflection of the more stringent scoring in Farran et al. In their study, if a participant looked down an incorrect path but didn't travel down it, or travelled any distance down a correct path this was scored as an error; in the current experiment, participants were not penalized for such behaviour. If participants with WS chose to look at their path choices before making a decision, this would explain the difference between the results of the current study and that of Farran et al. (2012a).

If route-learning is a relative strength for people with WS, one possibility is that they use their relative proficiency in language to facilitate a verbal strategy in performing the route-learning task, as they do to support number processing (Ansari, Donlan, Thomas, Ewing, Peen \& Karmiloff-Smith, 2003). Against this explanation, however, Farran et al. (2010) found evidence suggesting that people with WS do not spontaneously use a verbal strategy when learning a route. Another possibility is that people with WS might use good visual recognition skills to support route learning: there is evidence of chronological-age-appropriate face recognition ability in WS (Bellugi, Wang \& Jernigan, 1994; Wang, Doherty, Rourke \& Bellugi, 1995), which seems to be based on featural, rather than configural, processing (Karmiloff-Smith, 1997). This featural processing style applies to nonface stimuli in addition to faces (Karmiloff-Smith, Thomas, Annaz, Humphreys, Ewing, Grice, Brace, Van Duuren, Pike \& Campbell, 2004). This hypothesis also fits with the contrast between the current study and Farran et al. (2012a), which suggests that participants with WS look down correct and incorrect paths before making a decision more than TD children. This 'looking' behaviour is consistent with a strategy of looking at landmarks for recognition purposes, and thus using a visual recognition strategy rather than learning a route by recalling the sequence of landmarks and turns.

Experiment 2 was conducted to assess the ability to use each landmark type to successfully learn routes using mazes where only one type of landmark was available. We hypothesized that all participants would make fewer errors when path or junction landmarks only were available than when distant landmarks only were available.

\section{Experiment 2 Method}

\section{Participants}

Participants were the same as those in Experiment 1, apart from five of the DS group who were unavailable for testing: 108 TD individuals, 45 individuals with DS, and 19 individuals with WS. Experiment 2 was conducted five months after Experiment 1.

\section{Maze task}

There were three maze designs, each conforming to the specification given for Experiment 1. There were three conditions: a maze with junction landmarks only, a maze with path landmarks only, and one with distant landmarks only. The conditions were counterbalanced across the three maze designs (there were nine mazes in total). 
Each participant undertook three mazes, one for each condition, with order and maze design counterbalanced.

In the junction condition, there were 16 landmarks: eight at junctions on the correct paths, and eight at the ends of incorrect paths (that appeared to be at junctions when viewed from the correct path).

In the path condition, there were 16 landmarks: eight on the correct path, situated in the middle of path segments (away from junctions), and eight in the middle of incorrect path segments.

In the distant condition, there were just three large landmarks, positioned outside of the maze walls, with each visible from most locations within the maze.

As in Experiment 1 landmark objects were drawn from a range of categories and chosen for high verbal frequency (Morrison et al., 1997) and easy visual recognition.

\section{Procedure}

After participants completed the same familiarization maze as in Experiment 1, the experimenter showed them the correct route through the first experimental maze, saying to the participant, 'Pay close attention to the route and also to the various objects that appear in the "maze game" because you will have to go exactly the same way through the maze after I have shown you.'

The participant then attempted to navigate through the maze, with a maximum of three trials to complete the maze from start to finish without error. This maximum trial number was lower than that used in Experiment 1, and was chosen to prevent testing sessions from becoming too long for participants to complete. Errors were scored in the same way as in Experiment 1.

\section{Experiment 2 Results}

Descriptive statistics for route-learning and test battery items are given in Table 1. As in Experiment 1, the development of route learning was explored for each group, with respect to non-verbal ability.

A repeated-measures ANOVA of route-learning errors, with landmark type (junction, path and distant) as the repeated measure was run for each group. For the TD group, there was a main effect of landmark type, $F(2$, $214)=44.700, p<.001, \eta_{p}{ }^{2}=.295$; post-hoc Bonferroni corrected $t$-tests revealed more errors in the mazes with distant landmarks than both the mazes with junction landmarks, $p<.001$, and with path landmarks, $p<.001$, but no difference between performance on the mazes with path and junction landmarks, $p=.836$.
The DS group also showed a main effect of landmark type, $F(2,88)=5.721, p<.01, \eta_{p}{ }^{2}=.115$; they made more errors in mazes with distant landmarks than mazes with junction landmarks, $p<.01$, but there was no difference on distant and path mazes, $p=.238$, or junction and path mazes, $p=.293$

There was a main effect of landmark type for the WS group, $F(2,36)=3.759, p<.05, \eta_{p}{ }^{2}=.173$; however, the WS group made similar numbers of errors in distant mazes and junction mazes, $p=.302$, path and junction mazes, $p=.929$, and distant and path mazes, $p=.081$. The main effect is likely driven by marginal effect of more errors in the distant than the path maze.

Although the groups were not matched, it is worth noting that there was an effect of group, overall, $F(2$, 169) $=138.747, p<.001, \eta_{p}{ }^{2}=.451$, due to poorer performance by the DS group than the TD, $p<.001$, and WS, $p<.01$, groups, but with no difference between the WS and TD groups, $p=.932$.

Comparison between the numbers of errors made in the first three learning trials in Experiment 1 and performance in Experiment 2 (in which participants completed three learning trials per maze only) enables us to establish any detrimental effects of the presence of one landmark type (Experiment 2) relative to the full complement of landmark types (Experiment 1). ANOVA of maze type (Experiment 1, Junction, Path, Distant) by Group demonstrated a main effect of maze type, $F(3,507)$ $=17.528, p<.001, \eta_{p}{ }^{2}=.094$, which was consistent across groups, $F(6,507)=1.400, p=.212, \eta_{p}^{2}=.016$. Pairwise comparisons (Bonferroni corrected) demonstrated that the source of the main effect of maze was solely due to poor performance on the distant maze compared to other mazes ( $p<.001$ for all). Performance on the junction only and path only mazes did not differ from the full landmark maze used in Experiment 1 ( $p>.05$ for both).

Comparable performance on the junction and path landmark-only mazes with the Experiment 1 maze suggests that participants could learn these routes. To determine whether learning took place in the distant maze, comparison between participants' first and third learning trials was compared. Participants who made no errors on the first and second learning trials did not complete a third learning trial (they had met criterion) and so are not included in the analysis. This demonstrated a main effect of learning trial, $F(1,131)=4.52, p$ $=.035, \eta_{p}{ }^{2}=.033$, which did not interact with group, $F<$ 1 , thus demonstrating that learning did take place within the distant maze despite poor performance on this maze. A similar analysis of the TD group, split by age group (5-6 years, 7-8 years, 9-11 years), demonstrated the same pattern (Trial: $F(1,77)=7.21, p=.009, \eta_{p}^{2}=.086$; Trial by Group: $\mathrm{F}<1)$. 


\section{Trajectory analyses}

As in Experiment 1, ANCOVAs were conducted on TD and DS Maze Error with group as the between-subjects factor and RCPM as the covariate. The models included interaction terms between the RCPM covariate and the between-subjects factor. The data from the WS group were analysed separately, with a TD group individually matched on RCPM. This was because the data were positively skewed as a large proportion of the WS group made no Maze Errors.

\section{Mazes with junction landmarks}

As in Experiment 1, at the intercept the DS group's Maze Errors were significantly higher than the TD group's, $F(1,149)=8.97, p=.003, \eta_{p}{ }^{2}=.057$, and the relationship between RCPM and Maze Error was steeper in the DS group than the TD group, $F(1,149)=5.376, p$ $<.05, \eta_{p}{ }^{2}=.035$, i.e. the DS group 'catch up' with TD performance with increasing RCPM score (Figure 4).

The WS and TD groups did not significantly differ in Maze Errors, $U(38)=154.00, p=.407$.

\section{Mazes with path landmarks}

Again, at the intercept the DS group's Maze Errors were significantly higher than the TD group's, $F(1,149)=12.43$, $p=.001, \eta_{p}{ }^{2}=.077$, and the relationship between RCPM and Maze Error was steeper in the DS group than the TD group, $F(1,149)=9.597, p<.01, \eta_{p}{ }^{2}=.061$ (Figure 5).

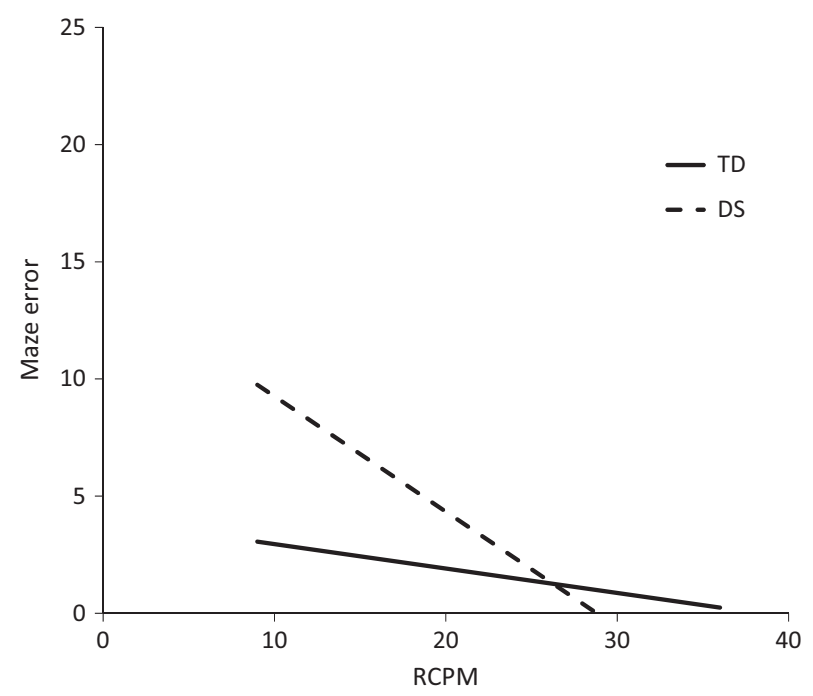

Figure 4 Experiment 2, mazes with junction landmarks: Maze Error against RCPM score for the TD and DS groups. Note: $T D=$ typically developing, DS = Down syndrome, $R C P M=$ Raven's Coloured Progressive Matrices.
The WS and TD groups did not differ in Maze Errors, $U(38)=159.00, p=.509$.

\section{Mazes with distant landmarks}

There was no group difference at the intercept, $F<1$, or in the relationship between RCPM and Maze Error for the DS group compared to the TD group, $F<1$ (Figure 6).

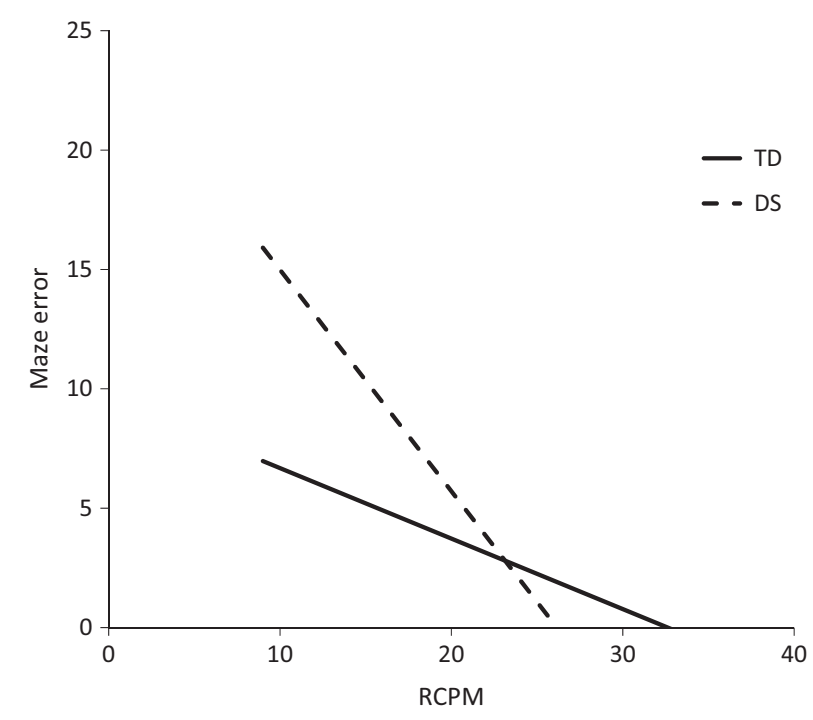

Figure 5 Experiment 2, mazes with path landmarks: Maze Error against RCPM score for the TD and DS groups. Note: $T D=$ typically developing, DS = Down syndrome, $R C P M=$ Raven's Coloured Progressive Matrices.

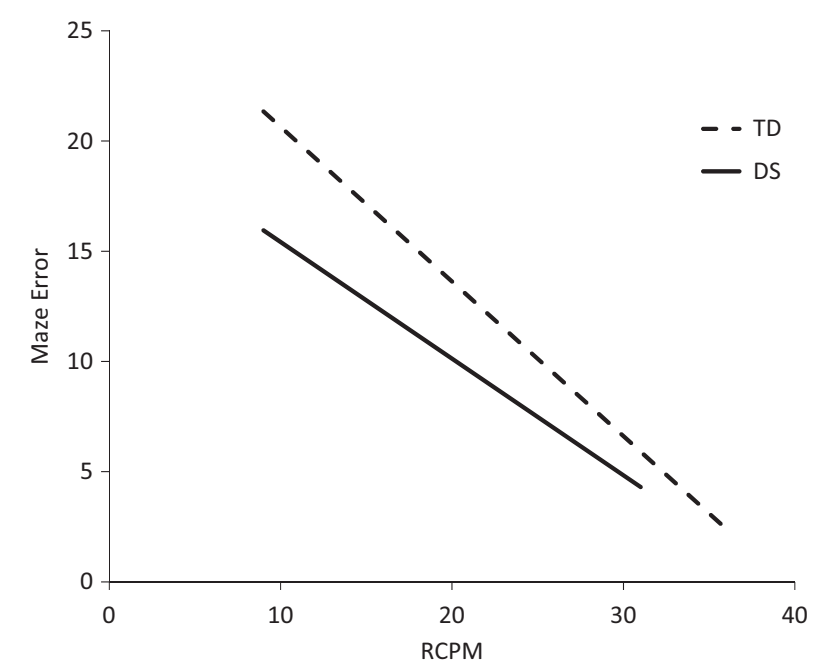

Figure 6 Experiment 2, mazes with distant landmarks: Maze Error against RCPM score for the TD and DS groups. Note: $T D=$ typically developing, DS = Down syndrome, $R C P M=$ Raven's Coloured Progressive Matrices. 
The WS group made fewer Maze Errors than the matched TD group, $U(38)=99.00, p=.017$.

\section{Cross-maze comparisons}

Repeated-measures ANCOVAs were conducted separately for the TD and DS groups, with landmark type as the within-subjects factor and RCPM as the covariate. Simple effects of task are independent of the covariate and have been reported in the ANOVAs above.

For the TD group, performance improved with RCPM, $F(1,106)=32.282, p<.001, \eta_{p}{ }^{2}=.233$. There was an interaction between RCPM and landmark type, $F(2,212)=7.770, p<.01, \eta_{p}{ }^{2}=.068$, reflecting a steeper relationship between RCPM and performance on distant mazes than both junction, $p<.05$, and path mazes, $p<.01$, and on path mazes compared with junction mazes, $p<.05$.

For the DS group, there was an improvement of performance with higher RCPM score, $F(1,43)=11.430$, $p<.01, \eta_{p}{ }^{2}=.210$. However, there was no reliable interaction between RCPM and landmark type, $F<1$.

\section{Experiment 2 Discussion}

The aim of Experiment 2 was to investigate the development of the ability to use different types of landmarks in typical development, Down syndrome and Williams syndrome. Because of the lower maximum trial number for each maze in Experiment 2, total errors were lower than for those observed in Experiment 1. However, comparison across experiments using equivalent numbers of learning trials demonstrated that participants were just as competent route learners when presented with just junction landmarks or just path landmarks, relative to a maze with all three landmark types. Consistent with Experiment 1, when compared across groups, the poorest performance was observed in the DS group. We first discuss the performance of the TD group before turning to the patterns of performance in the atypical groups.

The TD group's performance was better when junction or path landmarks were present than when only distant landmarks were available, but even the youngest children tested could learn using distant landmarks alone. However, there was no evidence for any overall advantage of junction landmarks over path landmarks in the TD group when the three age groups were examined separately. In line with the hypothesis that an advantage in using junction over path landmarks would emerge developmentally for the TD group, using a much higherpower analysis of the whole group, however, there was a difference in the relationship between non-verbal ability and performance across the path and junction conditions, with higher-ability individuals performing better in path mazes, relative to lower-ability individuals, than in junction mazes. In other words, development learning was 'faster' for junction landmark use than for path landmark use. This emphasizes the usefulness of trajectory analyses: only when performance was considered with respect to a developmental measure, RCPM, was an advantage with junction over path landmarks revealed (in line with the results of Farran et al., 2012a).

The 5- to 6-year-old TD children were able to successfully learn across the three learning trials when only distant landmarks were available, although with more errors in the process than when landmarks were present within the maze walls. Although it is possible that these children succeeded at the task without reference to the distant landmarks, simply by remembering the sequence of left and right turns, this seems unlikely for two reasons. First, there were six turns in each of the mazes, making it very difficult for the youngest children to remember the route as a verbal list, since 5-year-old children tend to have verbal memory spans of around three (increasing up to about five items by 11 years old; Hulme, Thomson, Muir \& Lawrence, 1984; Nicolson, 1981). Second, children younger than 7 years old do not spontaneously recode visually presented verbal material into a phonological form (e.g. Conrad, 1971; Henry, 1991; Hitch, Halliday, Dodd \& Littler, 1989). Given these points, along with the salience of the landmarks in an otherwise very sparse and repetitive brick wall environment, we conclude that even the youngest children tested were able to make use of distant landmarks. There was a steeper relationship between non-verbal ability and performance with distant landmarks over the other landmark types; considered together with the junction vs. path results, this indicates a developmental schedule in which junction landmarks can be used successfully earlier than path ones, which can both be used earlier than distant landmarks.

The DS group made more errors than the TD group overall. Initial analyses appeared to show a similar pattern of Maze Errors. That is, they made fewer routelearning errors in mazes with junction landmarks than in mazes with distant landmarks, but there were no differences in performance across the other maze types. Thus, people with Down syndrome are able to make better use of more useful landmarks, in a similar way to the TD sample in the study. However, for mazes with path or junction landmarks, the trajectory analyses showed different patterns for the DS and TD groups: in each case, better route-learning was associated with higher RCPM scores, but low non-verbal ability had 
more of a negative impact on route-learning performance in the DS group than in the TD group. This is the same pattern that was found for Experiment 1.

In mazes where only distant landmarks were visible, the DS trajectory was not significantly different from the TD trajectory, in line with our predictions. However, this result should be treated with caution because both groups' variability in Maze Error was high in this condition.

Across maze conditions, the pattern of results was different for the WS group from the TD and DS groups: there were no performance differences across the different maze types. Caution should be used in attempting to interpret null results, given the sample size of the WS, but the lack of differences in performance across the different maze types suggests that the WS group could have approached the task in a different way from the other groups. When compared with a subset of TD participants matched for non-verbal ability, the WS group performed better than the TD group only on the distant landmark maze. This may relate to a more featural visual processing style (Karmiloff-Smith, 1997), discussed further below.

\section{General discussion}

In Experiment 1, attention was a particularly strong predictor of route-learning ability across TD, DS and WS groups. In Experiment 2, the TD and DS groups showed the ability to use junction and path landmarks over distant landmarks, while there were no performance differences across landmark types for the WS group.

Trajectory analysis was possible for TD and DS groups only, for both experiments. This is unfortunate as it precluded developmental assessment of the WS group. Further research in which the task design elicits higher error rates in the WS group would enable trajectory analysis in this group. Despite this, we were still in a position to make cross-syndrome comparisons regarding absolute levels of ability on each of the four mazes, in addition to the patterns of performance across mazes.

Across both Experiments 1 and 2, the largest deficit in route learning was observed in the DS group, specifically those with low levels of non-verbal ability where routelearning performance was below that expected for their level of non-verbal ability. In contrast, performance of the WS group was in line with or above the level predicted by their level of non-verbal ability. Given that the two disorder groups had similar levels of non-verbal ability (and attentional control), this suggests that the WS group were able to draw on a compensatory strategy to boost their performance.
For the TD group only, an emerging ability to make better use of junction landmarks over path ones was demonstrated, with increasing non-verbal ability. This effect was subtle, however, indicating that path landmarks may be as useful as junction landmarks for TD children, at least in certain circumstances: e.g. when path landmarks are only a few seconds' walk away from a junction, and (critically) when an environment is relatively sparse. Strikingly, even 5- to 6-year-old TD children could use distant landmarks.

The WS group performed better than a matched TD subgroup on both Experiment 1, where junction, path, distant, and non-unique landmarks were all present, and also in Experiment 2, on mazes where only distant landmarks were present. This is a surprising result that contradicts our prediction that the WS group would perform at or below general levels of visuo-spatial ability on all route-learning tasks. What do these two maze types have in common that could explain this advantage of the WS group? Although more data are needed, we tentatively suggest that the particular challenge in each might be selecting relevant/useful foci for attention, or switching between them. In Experiment 1, the participant must choose which of the numerous landmarks to attend to at each time point; in the distant condition of Experiment 2, the participant must choose whether to attend to the landmarks outside of the maze or to the path/junction in view within the maze (not path or junction landmarks, but the path or junction itself). In such circumstances, the featural processing style associated with WS might enhance performance above that of participants with more holistic or global processing styles, who would attend to more irrelevant or less useful visual information at any given time. Karmiloff-Smith (1997) found that a WS group performed at chronological-age-appropriate level in a face recognition task that could be performed using a featural strategy. To the extent that maze performance in the current study requires featural processing, then, one might expect the WS group to perform better than the TD group. What might support good featural processing? Visual attention is an obvious candidate. In a review of attention in WS, Breckenridge, Atkinson and Braddick (2012) note that WS samples scored at a mental-age-appropriate level on the flanker task, which assessed selective attention, but below mental age level on a visual search task; inhibition and set shifting tasks. However, sustained attention was above mental age level. Given the importance of attention for route learning in WS found in the current study, an elucidating line of future research would be to explore directly the relationship between various types of attention and route learning in WS. 
Performance was in line with non-verbal ability in the DS group across the whole range of non-verbal ability only in the distant condition of Experiment 2. In all other conditions, route-learning performance was below the level predicted by non-verbal ability for individuals with low non-verbal scores (with performance 'catching up' at higher levels of non-verbal ability), but in the distant condition of Experiment 2 no such group difference was found. This further supports evidence for within-domain variability in DS; route learning for people with low nonverbal scores represents a relative weakness within the non-verbal domain. The present finding sits somewhere between the findings of Pennington et al. (2003) and Edgin et al. (2010) and demonstrates that poor nonverbal ability can be particularly detrimental to some aspects of route learning in this group. At this point, this puzzling pattern of results calls for more data: again, a thorough investigation of the relationship of different attentional abilities to route learning seems a promising avenue of research for DS, as for WS.

\section{Acknowledgements}

Thank you to Gauthier Billiet for help with data coding. Thank you to Bounds Green Primary School, the Williams Syndrome Foundation UK, and the Down's Syndrome Association, for enabling this research to take place. Special thanks go to the participants for taking part in the study. This research was supported by ESRC Grant RES-062-33-0005 and Agence Nationale de la Recherche (ANR) Grant ANR-09-FRBR-035.

\section{References}

Ansari, D., Donlan, C., Thomas, M.S.C., Ewing, S.A., Peen, T., \& Karmiloff-Smith, A. (2003). What makes counting count? Verbal and visuo-spatial contributions to typical and atypical number development. Journal of Experimental Child Psychology, 85, 50-62.

Baddeley, A.D., Emslie, H., \& Nimmo-Smith, I. (1994). Doors and people: A test of visual and verbal recall and recognition. Bury St Edmunds: Thames Valley Test Company.

Bellugi, U., Wang, P.P., \& Jernigan, T.L. (1994). Williams syndrome: an unusual neuropsychological profile. In $\mathrm{S}$. Broman \& J. Grafman (Eds.), Atypical cognitive deficits in developmental disorders: Implications for brain function (pp. 23-56). Hillsdale, NJ: Erlbaum.

Breckenridge, K., Atkinson, J., \& Braddick, O. (2012). Attention. In E.K. Farran \& A. Karmiloff-Smith (Eds.), Neurodevelopmental disorders across the lifespan: $A$ neuroconstructivist approach (pp. 119-134). Oxford: Oxford University Press.
Broadbent, H.J., Farran, E.K., \& Tolmie, A. (in press). Egocentric and allocentric navigation strategies in Williams syndrome and typical development. Developmental Science.

Chapman, R.S. (1995). Language development in children and adolescents with Down syndrome. In P. Fletcher \& B. MacWhinney (Eds.), Handbook of child language (pp. 641663). Oxford: Blackwell.

Conrad, R. (1971). The chronology of the development of covert speech in children. Developmental Psychology, 5, 398405.

Contestabile, A., Fila, T., Ceccarelli, C., Bonasoni, P., Bonapace, L., Santini, D., Bartesaghi, R., \& Ciani, E. (2007). Cell cycle alteration and decreased cell proliferation in the hippocampal dentate gyrus and in the neocortical germinal matrix of fetuses with Down syndrome and in Ts65Dn mice. Hippocampus, 17, 665-678.

Cornell, E.H., Heth, C.D., \& Broda, L.S. (1989). Children's way finding: response to instructions to use environmental landmarks. Developmental Psychology, 25, 755-764.

Doeller, C.F., King, J.A., \& Burgess, N. (2008). Parallel striatal and hippocampal systems for landmarks and boundaries in spatial memory. Proceedings of the National Academy of Sciences, USA, 105, 5915-5920.

Donnai, D., \& Karmiloff-Smith, A. (2000). Williams syndrome: from genotype through to the cognitive phenotype. American Journal of Medical Genetics, 97, 164-171.

Dunn, L.M., Dunn, D.M., Styles, B., \& Sewell, J. (2009). British Picture Vocabulary Scale III. Windsor: NFER-Nelson.

Dunn, L.M., Theriault-Whalen, C.M., \& Dunn, L.M. (1993). Echelle de Vocabulaire en Images Peabody. French adaptation of the Peabody Picture Vocabulary Test-Revised. Belgium: ATM.

Edgin, J.O., Mason, G., Allman, M., Capone, G., DeLeon, I., Maslen, C., Reeves, R.H., Sherman, S., \& Nadel, L. (2010). Development and validation of the Arizona Cognitive Test Battery for Down Syndrome. Journal of Neurodevelopmental Disorders, 2, 149-164.

Ewart, A.K., Morris, C.A., Atkinson, D., Jin, W., Sternes, K., Spallone, P., Stock, A.D., Leppert, M., \& Keating, M.T. (1993). Hemizygosity at the elastin locus in a developmental disorder, Williams syndrome. Nature Genetics, 5, 11-16.

Farran, E.K., Blades, M., Boucher, J., \& Tranter, L.J. (2010). How do individuals with Williams syndrome learn a route in a real world environment? Developmental Science, 13, 454 468.

Farran, E.K., Courbois, Y., Van Herwegen, J., \& Blades, M. (2012a). How useful are landmarks when learning a route in a virtual environment? Evidence from typical development and Williams syndrome. Journal of Experimental Child Psychology, 111, 571-586.

Farran, E.K., Courbois, Y., Van Herwegen, J., Cruickshank, A.G., \& Blades, M. (2012b). Colour as an environmental cue when learning a route in a virtual environment: typical and atypical development. Research in Developmental Disabilities, 33, 900-908.

Farran, E.K., Jarrold, C., \& Gathercole, S.E. (2003). Divided attention, selective attention and drawing: processing prefer- 
ences in Williams syndrome are dependent on the task administered. Neuropsychologia, 41, 676-687.

Gray, V., Karmiloff-Smith, A., Funnell, E., \& Tassabehji, M. (2006). In-depth analysis of spatial cognition in Williams syndrome: a critical assessment of the role of the LIMK1 gene. Neuropsychologia, 44, 679-685.

Henry, L.A. (1991). The effects of word length and phonemic similarity in young children's short-term memory. Quarterly Journal of Experimental Psychology, 43, 35-52.

Hitch, G.J., Halliday, M.S., Dodd, A., \& Littler, J.E. (1989). Development of rehearsal in short-term memory: differences between pictorial and spoken stimuli. British Journal of Developmental Psychology, 7, 347-363.

Howlin, P., Davies, M., \& Udwin, O. (1998). Cognitive functioning in adults with Williams syndrome. Journal of Child Psychology and Psychiatry, 39, 183-189.

Hulme, C., Thomson, N., Muir, C., \& Lawrence, A.L. (1984). Speech rate and the development of short-term memory span. Journal of Experimental Child Psychology, 38, 241-253.

Ishikawa, T., \& Montello, D.R. (2006). Spatial knowledge acquisition from direct experience in the environment: individual differences in the development of metric knowledge and the integration of separately learned places. Cognitive Psychology, 52, 93-129.

Jarrold, C., Baddeley, A.D., \& Phillips, C. (2007). Long-term memory for verbal and visual information in Down syndrome and Williams syndrome: performance on the Doors and People test. Cortex, 43, 233-247.

Jarrold, C., \& Brock, J. (2004). To match or not to match? Methodological issues in autism-related research. Journal of Autism and Developmental Disorders, 34, 81-86.

Karmiloff-Smith, A. (1997). Crucial differences between developmental cognitive neuroscience and adult neuropsychology. Developmental Neuropsychology, 13, 513-524.

Karmiloff-Smith, A. (1998). Development itself is the key to understanding developmental disorders. Trends in Cognitive Sciences, 2, 389-398.

Karmiloff-Smith, A., Thomas, M.S.C., Annaz, D., Humphreys, K., Ewing, S., Grice, S., Brace, N., Van Duuren, M., Pike, G., \& Campbell, R. (2004). Exploring the Williams syndrome face processing debate: the importance of building developmental trajectories. Journal of Child Psychology and Psychiatry and Allied Disciplines, 45, 1258-1274.

Lenhoff, H.M., Wang, P.P., Greenberg, F., \& Bellugi, U. (1997). Williams syndrome and the brain. Scientific American, 277, $42-47$.

Lott, I.T., \& Head, E. (2001). Down syndrome and Alzheimer's disease: a link between development and aging. Mental Retardation and Developmental Disabilities Research Reviews, 7, 172-178.

Meng, Y., Zhang, Y., Tregoubov, V., Janus, C., Cruz, L., Jackson, M., Lu, W.-Y., MacDonald, J.F., Wang, J.Y., Falls, D.L., \& Jia, Z. (2002). Abnormal spine morphology and enhanced LTP in LIMK-1 knockout mice. Neuron, 35, 121-133.

Menghini, D., Addona, F., Costanzo, F., \& Vicari, S. (2010). Executive functions in individuals with Williams syndrome. Journal of Intellectual Disability Research, 54, 418-432.

Meyer-Lindenberg, A., Hariri, A.R., Munoz, K.E., Mervis, C.B., Mattay, V.S., Morris, C.A., \& Berman, K.F. (2005). Neural correlates of genetically abnormal social cognition in Williams syndrome. Nature Neuroscience, 8, 991-993.

Montello, D.R. (1998). A new framework for understanding the acquisition of spatial knowledge in large-scale environments. In M.J. Egenhofer \& R.G. Golledge (Eds.), Spatial and temporal reasoning in geographic information systems (pp. 143-154). New York: Oxford University Press.

Morris, C.A., Dempsey, S.A., Leonard, C.O., Dilts, C., \& Blackburn, B.L. (1988). Natural history of Williams syndrome: physical characteristics. Journal of Paediatric Medicine, 113, 318-326.

Morris, R.G.M. (1981). Spatial localization does not require the presence of local cues. Learning and Motivation, 12, 239 260.

Morrison, C.M., Chappell, T.D., \& Ellis, A.W. (1997). Age of acquisition norms for a large set of object names and their relation to adult estimates and other variables. Quarterly Journal of Experimental Psychology, 50, 528-559.

Nicolson, R.I. (1981). The relationship between memory span and processing speed. In M. Friedman, J.P. Das \& N. O'Connor (Eds.), Intelligence and learning (pp. 179-183). London: Plenum.

Pennington, B.F., Moon, J., Edgin, J., Stedron, J., \& Nadel, L. (2003). The neuropsychology of Down syndrome: evidence for hippocampal dysfunction. Child Development, 74, 75-93.

Pinter, J.D., Brown, W.E., Eliez, S., Schmitt, J.E., Capone, G.T., \& Reiss, A.L. (2001). Amygdala and hippocampal volumes in children with Down syndrome: a high-resolution MRI study. Neurology, 56, 972-974.

Purser, H.R.M., Farran, E.K., Courbois, Y., Lemahieu, A., Sockeel, P., \& Blades, M. (2012). Short-term memory, executive control and children's route learning. Journal of Experimental Child Psychology, 113, 273-285.

Purser, H.R.M., \& Jarrold, C. (2005). Impaired verbal shortterm memory in Down syndrome reflects a capacity limitation rather than atypically rapid forgetting. Journal of Experimental Child Psychology, 91, 1-23.

Raven, J., Raven, J.C., \& Court, J.H. (1998). Coloured Progressive Matrices. Oxford: Oxford University Press.

Redick, T.S., Calvo, A., Gay, C.E., \& Engle, R.W. (2011). Working memory capacity and go/no-go task performance: selective effects of updating, maintenance, and inhibition. Journal of Experimental Psychology: Learning, Memory, and Cognition, 37, 308-324.

Siegel, A.W., \& White, S.H. (1975). The development of spatial representations of large-scale environments. In H.W. Reese (Ed.), Advances in child development and behavior, Vol. 10 (pp. 9-55). New York: Academic Press.

Steele, J., \& Stratford, B. (1995). The United Kingdom population with Down syndrome: present and future projections. American Journal on Mental Retardation, 99, 664-682.

Thomas, M.S.C., Annaz, D., Ansari, D., Scerif, G., Jarrold, C., \& Karmiloff-Smith, A. (2009). Using developmental 
trajectories to understand developmental disorders. Journal of Speech, Language, and Hearing Research, 52, 336-358.

Van Herwegen, J., Rundblad, G., Davelaar, E.J., \& Annaz, D. (2011). Variability and standardized test profiles in typically developing children and children with Williams syndrome. British Journal of Developmental Psychology, 29, 883-894.

Wang, P.P., Doherty, S., Rourke, S.B., \& Bellugi, U. (1995). Unique profile of visuo-spatial skills in a genetic syndrome. Brain and Cognition, 29, 54-65.
Wegman, J., \& Janzen, G. (2011). Neural encoding of objects relevant for navigation and resting state correlations with navigational ability. Journal of Cognitive Neuroscience, 23, 3841-3854.

Received: 6 May 2014

Accepted: 15 July 2014 